\title{
Fatty Acid Composition of Eucommia Rubber Particles, Content and Molecular Weight Distribution of Eu-rubber in Eucommia Ulmoides Leaves and Samaras
}

\author{
Li-Jun Qin \\ Guizhou University \\ Ting Yuan ( $\nabla$ yuantliss@163.com ) \\ Guizhou University https://orcid.org/0000-0003-1488-4073 \\ ZhengWei Yang \\ Guizhou University \\ DeGang Zhao \\ Guizhou University
}

\section{Original Article}

Keywords: Eucommia ulmoides, rubber particles, fatty acids, molecular weight

Posted Date: October 14th, 2020

DOI: https://doi.org/10.21203/rs.3.rs-89690/v1

License: (c) (1) This work is licensed under a Creative Commons Attribution 4.0 International License.

Read Full License 
Fatty acid composition of Eucommia rubber particles, content and molecular weight

distribution of Eu-rubber in Eucommia ulmoides leaves and samaras

\author{
Li-Jun Qin ${ }^{\mathrm{a}, \mathrm{b}^{*}}$, Yuan Ting ${ }^{\mathrm{b} *}$, Yang Zhengwei ${ }^{\mathrm{b}}$, De-Gang Zhao ${ }^{\mathrm{a}, \mathrm{c}}{ }^{\S}$
}

${ }^{a}$ The Key Laboratory of Plant Resources Conservation and Germplasm Innovation in Mountainous Region (Ministry of Education), Guizhou University, Guiyang, Guizhou Province, PR China

${ }^{b}$ Key Laboratory of Plant Resource Conservation and Germplasm Innovation in Mountainous Region (Ministry of Education), Collaborative Innovation Center for Mountain Ecology \& AgroBioengineering (CICMEAB), College of Life Sciences/Institute of Agro-bioengineering, Guizhou University, Guiyang 550025, Guizhou Province, China

${ }^{\mathrm{c}}$ Guizhou Academy of Agricultural Sciences, Guiyang 550006, PR China

Abstract: Abstact: [Backgroud] Eucommia ulmoides rubber particles (EuRPs) wrapped by a monolayer protein-lipid membrane are speculated to an important site for the biosynthesis of $E$. ulmoides rubber (Eu-rubber), and the types and contents of these protein and lipid varied in different E. ulmoides organs which might indicate the difference of Eu-rubber biosynthesis in EuRPs from different organs and tissues. In this paper, the morphological structure and fatty acid composition of EuRPs in leaves and samaras collected in October, 2019 were compared, and molecular weight (Mw) and molecular weight distribution (MWD) were also analyed by GPC system. [Results] The results showed that small rubber particles (SRPs, 1 4 $\mu \mathrm{m}$ ) and medium rubber particles (MRPs, $4 \sim 7 \mu \mathrm{m}$ ) from leaves and samaras respectively accounted for 39\%, 53\% and 38\%, 34\%, while the amount of the large rubber particles (LRPs, 7 11 $\mu \mathrm{m}$ ) were $23 \%$ and 13\%, which indicated there was no significance in leaf and samara SRPs while the amount of MRPs and LRPs in leaf both were more than that of in samara. Synchronously, the determination results of fatty acids (FAs) compositon showed three of the 35 kinds FAs were uniquely detected in leaf rubber particles (LeRPs), which included eleven carbonic (C11:0), thirteen carbonic (C13:0) and arachidic acids (C20:0), while $\gamma$ linolenic acid (C18:3n6) was exclusively found in samara rubber particles (SaRPs) with a lower content. Additioanlly, five characteristic distribution peaks (CDPs) of Eu-rubber from leaves were detected while there were only 2-3 CDPs in samaras; Further, the MWD analysis suggested that the MWD of Eu-rubber in E. ulmoides samaras and leaves were 40.61 and 6.76 respectively, which indicated the more Eu-rubber molecules with various $\mathrm{Mw}$ existed in samara compared with those in leaf. [Conclusions] Together, we concluded that average size of LeRPs was bigger than SaRPs in October, and 3 kinds of saturated fatty acid (SAF) were unique to the leaves while only one of unsaturated fatty acid (UFA) was sole to SaRPs. Simultaneously, the Eu-rubber in samara in October possessed more rubber molecules with various molecular weights compared with the contemporaneous leaves.

Keywords: Eucommia ulmoides; rubber particles; fatty acids; molecular weight

\title{
Introduction
}

Eucommia ulmoides Oliver ('Duzhong' in Chinese; 'Tuchong' in Japanese), the single extant species of Eucommiaceae, is a dioecious, wind-pollinated tree diffusely distributed in the mixed mesophytic forest habitats of valleys, hills, and low mountains in central and eastern China (Cronquist 1981; Zhang et al. 2003). Duzhong is not only a valuably Chinese herbal medicines with 
the pharmacological functions to treat lumbago and hypertension, prevent bone senescence, and to enhance liver and kidney functions (Chinese Pharmacopoeia Committee 1990), but it has also been used as an important ru bber tree species(Bamba et al. 2001), especially as a natural substitute for petroleum-based synthetic rubber (PBSR). E. ulmoides rubber (EU-rubber), as a green renewable material, has been applied in the chemical industry medicine field as a raw material (Sun et al. 2013; Dong et al. 2011). EU-rubber is mainly composed of trans-1,4-polyisoprene (TPI), which is the isomer of natural rubber (NR), a cis-1,4-polyisoprene (CPI) (van Beilen and Poirier 2007; Zhang and Xue 2011). Previous studies reported rubber particles (RPs), including large rubber particles (LRPs) and small rubber particles (SRPs), were both the sites of NR biosynthesis (Nawamawat et al. 2011).

The results based on transmission electron microscopy (TEM) of tissue sections of Hevea brasiliensis shows that the particles consist of an apparently homogeneous core surrounded by a contiguous boundary, with the core appearing somewhat less electron-dense than the boundary, which implied the structure of the rubber (polyisoprene)-containing cores surrounded by biomembrane of non-rubber components (NRCs, e.g. proteins and phospholipids, etc) (de Fay et al. 1989; Wu et al. 2014). Actually, the further studies proves that there are neutral lipids accounting for $2.4 \%$, glycolipids and phospholipids for $1.0 \%$, proteins for $2.2 \%$, carbohydrates for $0.4 \%$, ash for $0.2 \%$, and other compounds for $0.1 \%$ in the commercial solid NR (Nishiyama et al. 1996). These NRCs are suspected of being responsible for both the superior properties and the inconsistent quality of NR (Chollakup et al. 2013). For instance, the whole lipid content have been reported to be inversely proportional to the tack properties of NR (David et al. 2001) while unsaturated fatty acids (UFAs) were reported to act as a plasticizer for rubber by lowering the plasticity of NR (Kakubo et al. 1997). On the contrary, some saturated fatty acids (SFAs) were found to affect NR crystallization by acting as a nucleating agent, which improved characteristic NR green strength in the unvulcanized state (Kawahara et al. 1999). As reported previously, the NR molecules chain consisting of $\omega$-terminal and $\alpha$-terminal, has been postulated to link with mono-phosphate and diphosphate groups associated with phospholipids by H-bonding at the $\alpha$-terminal, and the $\omega$-terminal is a dimethylallyl group linked to the protein by H-bonding ( $\mathrm{Wu}$ et al. 2014). For the past few years, a series of evidence suggested that the $\omega$-terminus bonds with a peptide or unidentified functional group, whereas the $\alpha$-terminus consists of phosphoric ester and two long-chain fatty acid ester groups (Tarachiwin et al. 2005a, Tarachiwin et al. 2005b, Tarachiwin et al. 2005), which implys that the long chain fatty acid esters may be a constituent of the $\alpha$-terminal group in NR chain that is expected to link with the rubber molecule as a component of a glyceride or phospholipid (Cornish 2001). Hence, the kind and content of fatty acids will be certainly to affect the properties of NR, e.g. molecular weight $(\mathrm{Mw})$, molecular weight distribution (MWD), etc. Although, numerous studies around the biosynthesis of EU-rubber have been conducted, there are few reports on morphological structure and NRCs of E. ulmoides rubber paricles (EuRPs) because of the low extraction rate and complex steps of separation and purification of EuRPs. Therefore, it is of great significance to analyze NRCs (especially fatty acids, FAs) constitute of EuRPs and Mw of Eu-rubber, and it will provide a theoretical basis for further elucidating the morphological structure of EuRPs, proving the relationships of FAs content and kind with molecular characteristics of Eu-rubber.

\section{Materials and methods}




\section{Plant Materials and Reagents}

Leaf and samara samples were collected randomly from 15 E. ulmoides clones (10 years old) with two or three plants per clone in a plantation of Guizhou University, and they were collected in October. EuRPs extracted from leaf or samara of E. ulmoides were stored in $-80^{\circ} \mathrm{C}$ for analysis of FAs and molecular weight of Eu-rubber. Trimethylolamine (Tris), phenyl-methylsulfonyl fluoride (PMSF), polyvinylpyrrolidone (PVP), $\beta$-mercaptoethanol (BME) and ascorbic acid (Vc) were purchased from Sigma-Aldrich LLC.; Potassium fluoride, magnesium sulfate, isopropanol and hydrochloric acid were purchased from Tianjin Kemiou Chemical Reagent Co., Ltd. Thirty-five standards of FAs were selected based on their different chain length, geometric structure (cis and trans) and double bond positions. The individual FAMEs were purchased from Fluka ( $>99 \%$, SigmaAldrich). All solvents and reagents were of an analytical grade, specially for chromatography and purchased from Systerm (Systerm, Malaysia) except n-hexane, which was of a higher purity grade (Systerm, Malaysia, for GC, P99\%). The esterifying agent TMS-DM (2 M) in $n$-hexane was purchased from Sigma (Sigma-Aldrich). Tetrahydrofuran (THF, chromatographically pure) and six mixed standards (PStQuick MP-M, Mw=706000, 96400, 5970, 474, 370 and 266 respectively) were purchased from TOSOH co. (Japan).

\section{Extraction and purification of EuRPs}

The EuRPs extraction were performed essentially as described by Yang et al. (2019) and Zhao (1999) with some modifications. The fresh leaf or samara remaining in $-80^{\circ} \mathrm{C}$ ultra-low temperature freezer was rapidly grinded into powder and took $100 \mathrm{~g}$ of powder sample into a $1 \mathrm{~L}$-flask containing $400 \mathrm{~mL}$ of the extract buffer $\left[100 \mathrm{mmol} \cdot \mathrm{L}^{-1}\right.$ Tris $-\mathrm{HCl}, 50 \mathrm{mmol} \cdot \mathrm{L}^{-1} \mathrm{KF}, 2 \%(\mathrm{~W} / \mathrm{V}) \mathrm{Vc}, 5 \mathrm{mmol} \cdot \mathrm{L}^{-1}$ $\mathrm{MgSO}_{4}, \mathrm{pH}=7.5$ ). adding $2 \%(\mathrm{~W} / \mathrm{V}) \mathrm{PVP}$ before use, $\beta$-mercaptoethanol (final concentration is 5 $\left.\mathrm{mmol} \cdot \mathrm{L}^{-1}\right), 0.1 \mathrm{mmol} \cdot \mathrm{L}^{-1} \mathrm{PMSF} /$ isopropanol solution] with rapid stirring extraction in the ice $1 \mathrm{~min}$. After that, the filtrate from the sample homogenate through 200 mesh stainless steel screen under the condition of $4^{\circ} \mathrm{C}$ was collected for later use. The filtrate was further divided into six centrifuge tubes $\left(50 \mathrm{~mL} \cdot\right.$ tube $\left.^{-1}\right)$ and the filtrate-containing tubes were centrifuged under the condition of $4^{\circ} \mathrm{C}$ for $10 \mathrm{~min}$ at $5000 \mathrm{~g}$ with fixed-angle rotors ( 45 degrees) and the centrifuge tubes were gently took out. The liquid supernatant was orderly transferred to numbers of $10 \mathrm{~mL}$ centrifuge tubes by a 20 $\mathrm{mL}$ clean syringe for further centrifugation at $5000 \mathrm{~g}$ for $8 \mathrm{~min}$ below $4^{\circ} \mathrm{C}$. The suspended solids on the top of liquid was then transferred to numbers of new $10 \mathrm{~mL}$ centrifuge tubes in which $8 \mathrm{~mL}$ of the rinsing solution was added in advance. The purification operation was performed by horizontal centrifugation of resuspension solution $\left[100 \mathrm{mmol} \cdot \mathrm{L}^{-1}\right.$ Tris- $\mathrm{HCl}, 5 \mathrm{mmol} \cdot \mathrm{L}^{-1} \mathrm{MgSO}_{4}, 10 \mathrm{mmol} \cdot \mathrm{L}^{-1}$ $\beta$-mercaptoethanol] at $5000 \mathrm{~g}$ for $8 \mathrm{~min}$ under the condition of $4^{\circ} \mathrm{C}$ and then remove rinsing solution, repeating the step of resuspendance and centrifugation 6-8 times. Purified EuRPs were stored in buffered $10 \%$ or $30 \%$ glycerol at $-20^{\circ} \mathrm{C}$ until prepared for microscopy.

\section{Microscopic observation of EuRPs}

Taking an appropriate amount (approx. $0.002 \mathrm{~g}$ ) of EuRPs samples from E. ulmoides leaves and samaras in October on a clean glass slide, the samples were placed on a clean glass slide, and 1 drop of resuspension buffer was added to suspend the rubber particles. The cover glass was placed under Olympus BX43 optical microscope (Japan, 1000×) to observe the morphology of EuRPs. Meanwhile, the measuring system build-in OLYMPUS BX43 was used to measure the particle size of the EuRPs in the sample material collected from October, and the EuRPs observed were classified 
and counted according to the particle size. The shape and diameter of rubber particles were determined by scanning electron microscopy (SEM) method refered by Singh et al. (2003). A drop of a suspension of EuRPs in glycerol was fixed in 3\% glutaraldehyde in $50 \mathrm{mM}$ sodium cacodylate buffer (containing $1 \%$ tannic acid) for $1 \mathrm{~h}$ at room temperature. After washing in several changes of buffer, the samples were post-fixed in $1 \%$ aqueous osmium tetroxide also for $1 \mathrm{~h}$ at room temperature, and subsequently washed in several changes of distilled water. A small drop of the suspension (appropriately diluted) was air-dried on a piece of cover glass. The cover glass was then placed on a stub applied with a double-sided tape, sputter-coated with gold and examined with a Hitachi S530 SEM at $10 \mathrm{kV}$.

\section{Determination of FAs in EuRPs}

The extraction of FAs from EuRPs was conducted by referring to Kittigowittana et al. (2016) with some modifications. Take $80-100 \mathrm{mg}$ ordinary sample of purified EuRPs into $15 \mathrm{~mL}$ centrifuge tube, and add $100 \mu \mathrm{L}$ methyl nonadecanoate as internal standard. Continue to add $2 \mathrm{~mL}$ of $2 \%$ sodium hydroxide methanol solution for $30 \mathrm{~min}$ water bath at $85^{\circ} \mathrm{C}$ in water bath kettle, add $3 \mathrm{~mL} 14 \%$ boron trifluoride $\left(\mathrm{BF}_{3}\right) /$ methanol solution for treatment under the same condition. After the temperature was reduced to room temperature, extra $1 \mathrm{~mL} n$-hexane was added into the centrifugal tube and the sample was extracted by shock for $2 \mathrm{~min}$, then left for $1 \mathrm{~h}$ to wait for stratification. Take $100 \mu \mathrm{L}$ of supernatant solution and allow to $1 \mathrm{~mL}$ with $n$-hexane. Use $0.45 \mu \mathrm{m}$ filter membrane to pass the membrane and then test on the machine. In addition, take 30-50 mg of the grease sample and dissolve it in ethanol. And the processing steps are the same as above. After the extraction process, FAMEs were prepared before the GC analysis. One microlitre volume of each sample was injected into GC systems (Trace1310-ISQ, Thermo Fisher Scientific, USA) equipped with FID for separation and quantification of the FAMEs. The analysis was carried out using a TG-5MS fused silica capillary column ( $30 \mathrm{~m}, 0.25 \mathrm{~mm}$ i.d., $0.25 \mu \mathrm{m}$ film thickness, Thermo Fisher Scientific, USA). The run was under an optimised temperature programme as follows: initial column temperature $80^{\circ} \mathrm{C}$ for $1 \mathrm{~min}$, programmed to increase at a rate of $10^{\circ} \mathrm{C} \cdot \mathrm{min}^{-1}$ up to $200^{\circ} \mathrm{C}$ and then at $5^{\circ} \mathrm{C} \cdot \mathrm{min}^{-1}$ up to $250^{\circ} \mathrm{C}$. Then at $2^{\circ} \mathrm{C} \cdot \mathrm{min}^{-1}$ up to final temperature of $270^{\circ} \mathrm{C}$ and held for $3 \mathrm{~min}$. Injector and detector temperatures were at $290^{\circ} \mathrm{C}$. Helium as used as the carrier gas at a flow rate of $1.2 \mathrm{~mL} \cdot \mathrm{min}^{-1}$ with a split ratio of 30:1. The contents of fatty acids in the samples were calculated according to formula (1) :

\section{$\underline{\text { Asi } \times \text { mskli } \times \mathrm{Fj}} \quad$ formula 1 \\ Astdi $\times \mathrm{m}$}

Where $\mathrm{X}_{\mathrm{i}}$ represents the amount of each fatty acid in the sample in milligrams per hundred grams $\left(\mathrm{mg} \cdot \mathrm{Kg}^{-1}\right) ; \mathrm{A}_{\mathrm{si}}$ represents the peak area of fatty acids in the liquid was determined; $\mathrm{m}_{\text {stdi }}$ represents the quality, in milligrams ( $\mathrm{mg}$ ), of the standard substance contained in the standard working solution of triglycerides of fatty acids extracted from the preparation of the standard determination solution; $F_{j}$ represents conversion coefficients of triglycerides of fatty acids to fatty acids (see table 1 ); $A_{\text {stdi }}$ represents standard determination of the peak area of fatty acids in liquid; $m$ represents the weight of the sample in grams $(\mathrm{kg})$. The arithmetic mean of two independent measurements obtained under repeatable conditions was expressed, and three significant digits were reserved for recording.

Table 1 A list of fatty acid types, detection limits and conversion coefficients of fatty acid methyl esters or triglycerides into fatty acids 


\begin{tabular}{|c|c|c|c|c|c|}
\hline \multirow{2}{*}{$\begin{array}{c}\begin{array}{c}\text { Serial } \\
\text { number }\end{array} \\
1\end{array}$} & \multicolumn{2}{|c|}{ Fatty acid name } & \multirow{2}{*}{$\begin{array}{c}\begin{array}{c}\text { Detection limit } \\
\left(\mathbf{m g} \cdot \mathbf{K g}^{-1}\right)\end{array} \\
0.5\end{array}$} & \multirow{2}{*}{$\begin{array}{c}\text { Fi conversion } \\
\text { coefficient }\end{array}$} & \multirow{2}{*}{$\begin{array}{c}\text { Fj conversion } \\
\text { coefficient }\end{array}$} \\
\hline & Octanoic acid & $\mathrm{C} 8: 0$ & & & \\
\hline 2 & Decanoic acid & $\mathrm{C} 10: 0$ & 0.5 & 0.9247 & 0.9314 \\
\hline 3 & Eleven carbonic acid & $\mathrm{C} 11: 0$ & 0.5 & 0.9300 & 0.9363 \\
\hline 4 & Lauric acid & $\mathrm{C} 12: 0$ & 0.5 & 0.9346 & 0.9405 \\
\hline 5 & Thirteen carbonic acid & $\mathrm{C} 13: 0$ & 0.5 & 0.9386 & 0.9442 \\
\hline 6 & Myristic acid & $\mathrm{C} 14: 0$ & 0.5 & 0.9421 & 0.9473 \\
\hline 7 & Myristoleic acid & $\mathrm{C} 14: \ln 5$ & 0.5 & 0.9417 & 0.9470 \\
\hline 8 & Fifteen carbonic acid & $\mathrm{C} 15: 0$ & 0.5 & 0.9453 & 0.9502 \\
\hline 9 & Hexadecanoic acid & $\mathrm{C} 15: \ln 5$ & 0.5 & 0.9449 & 0.9499 \\
\hline 10 & Palmitic acid & $\mathrm{C} 16: 0$ & 0.5 & 0.9481 & 0.9529 \\
\hline 11 & Palmitoleic acid & $\mathrm{C} 16: \ln 7$ & 0.5 & 0.9477 & 0.9525 \\
\hline 12 & Seventeen carbonic acid & $\mathrm{C} 17: 0$ & 0.5 & 0.9507 & 0.9552 \\
\hline 13 & Heptadecanoic acid & $\mathrm{C} 17: \ln 7$ & 0.5 & 0.9503 & 0.9549 \\
\hline 14 & Stearic acid & $\mathrm{C} 18: 0$ & 0.5 & 0.9530 & 0.9573 \\
\hline 15 & Elaidic acid & $\mathrm{C} 18: \ln 9 \mathrm{t}$ & 0.5 & 0.9527 & 0.9570 \\
\hline 16 & Oleic acid & $\mathrm{C} 18 \mathrm{~s} \ln 9 \mathrm{c}$ & 0.5 & 0.9527 & 0.9571 \\
\hline 17 & Linoelaidic acid & $\mathrm{C} 18: 2 \mathrm{n} 6 \mathrm{t}$ & 0.5 & 0.9524 & 0.9568 \\
\hline 18 & Linoleic acid & $\mathrm{C} 18 \mathrm{an} 6 \mathrm{c}$ & 0.5 & 0.9524 & 0.9568 \\
\hline 19 & Arachidic acid & $\mathrm{C} 20: 0$ & 0.5 & 0.9570 & 0.9609 \\
\hline 20 & $\gamma$-linolenic acid & C18:3n6 & 0.5 & 0.9520 & 0.9559 \\
\hline 21 & Eicosanoic acid & $\mathrm{C} 20: 1$ & 0.5 & 0.9568 & 0.9608 \\
\hline 22 & $\alpha$-linolenic acid & $\mathrm{C} 18: 3 \mathrm{n} 3$ & 0.5 & 0.9520 & 0.9560 \\
\hline 23 & Twenty-one carbonic acid & $\mathrm{C} 21: 0$ & 0.5 & 0.9588 & 0.9628 \\
\hline 24 & Eicosadienoic acid & $\mathrm{C} 20: 2$ & 0.5 & 0.9565 & 0.9605 \\
\hline 25 & Twenty-two carbonic acid & $\mathrm{C} 22: 0$ & 0.5 & 0.9604 & 0.9642 \\
\hline 26 & Eicotrienoic acid & C20:3n6 & 0.5 & 0.9562 & 0.9598 \\
\hline 27 & Erucic acid & $\mathrm{C} 22: \ln 9$ & 0.5 & 0.9602 & 0.9639 \\
\hline 28 & Eicotrienoic acid & $\mathrm{C} 20: 3 \mathrm{n} 3$ & 0.5 & 0.9562 & 0.9598 \\
\hline 29 & Arachidonic acid & C20:4n6 & 0.5 & 0.9560 & 0.9597 \\
\hline 30 & Tricosanoic acid & $\mathrm{C} 23: 0$ & 0.5 & 0.9620 & 0.9658 \\
\hline 31 & Docosadienoic acid & $\mathrm{C} 22: 2 \mathrm{n} 6$ & 0.5 & 0.9600 & 0.9638 \\
\hline 32 & Twenty-four carbonic acid & $\mathrm{C} 24: 0$ & 0.5 & 0.9963 & 1.0002 \\
\hline 33 & Timnodonic acid & $\mathrm{C} 20: 5 \mathrm{n} 3$ & 1.0 & 0.9557 & 0.9592 \\
\hline 34 & Tetracotenoic acid & C24:1n9 & 1.0 & 0.9632 & 0.9666 \\
\hline 35 & Ethyl Docosahexaenoate & $\mathrm{C} 22: 6 \mathrm{n} 3$ & 1.0 & 0.9590 & 0.9624 \\
\hline
\end{tabular}

Note $1 \mathrm{Fi}$ is the conversion coefficient of fatty acid methyl ester into fatty acid; Note $2 \mathrm{Fj}$ is the coefficient of conversion of fatty acid triglycerides to fatty acids.

\subsection{Mw Determine of EU-rubber}

Each EuRPs from leaves and samara were respectively dissolved in tetrahydrofuran (THF) to a concentration of $1.0 \mathrm{mg} / \mathrm{mL}$, incubated at $50^{\circ} \mathrm{C}$ overnight to allow for full dissolution and then filtered through a $0.22 \mu \mathrm{m}$ polytetrafluoroethylene (PTFE) syringe filter. The molecular-weight and 
moleeuiar-weight distribution of EU-rubber were measured by gel permeation chromatography (EcoSEC system, TOSOH, Japan) equipped with an HLC-8320GPC controller, auto-injector (1 to $1,500 \mu \mathrm{L}$ in $1 \mu \mathrm{L}$ increments), column oven (ambient plus $10^{\circ} \mathrm{C}$ to $60^{\circ} \mathrm{C}$ ), and an Bryce RI detector (tungsten light source, 1.00-1.80 RI range). The GPC was carried out using semi-micro SEC column (TSK-GEL SuperMultiporeHZ series) and PStQuick polystyrene standard kit was prepared as standard sample for minimum error. Sample volumes of $20 \mu \mathrm{L}$ were passed through an TSK chromatographic column $(4.6 \mathrm{IDmm} \times 150 \mathrm{~mm}, 3 \mu \mathrm{m}$ particle size, no. 21488) with the column oven maintained at $40{ }^{\circ} \mathrm{C}$. The mobile phase was THF at a flow rate of $0.35 \mathrm{~mL} / \mathrm{min}$. The weight average molecular-weight and the molecular-weight distribution were obtained with EcoSEC-WS work station.

\subsection{Statistical analysis}

All results were obtained from three independent experiments and expressed as mean $\pm \mathrm{SD}$. Analyses of variance were perfonned by ANOVA procedures and significant differences $(p<0.05)$ were determined by T test with SPSS Statistics18.0.

\section{Results and discussion}

\section{Comparison of the morphological characteristics of EuRPs}

The results of optical microscopy and scanning electron microscopy showed that the EuRPs were spherical and the shapes of the particles were different in different organs. In leaves of E. ulmoides, the content of small rubber particles (SRPs) (1-4 $\mu \mathrm{m})$ and medium rubber particles (MRPs) (4-7 $\mu \mathrm{m})$ were equal, accounting for $39 \%$ and $38 \%$ of the total EU-rubber particles respectively, and that of large rubber particles (LRPs) $(7-11 \mu \mathrm{m})$ accounted for $23 \%$. In the samara of E. ulmoides, the content of SRPs $(1-4 \mu \mathrm{m})$ was the highest, accounting for $53 \%$, the content of MRPs $(4-7 \mu \mathrm{m})$ was $34 \%$, and the content of LRPs was the lowest, accounting for 13\% (Fig. 1). The above results indicated that the size of rubber particles in different organs and tissues of E. ulmoides showed high specificity, and the difference in particle size of this kind of rubber particles may be related to the synthesis of rubber molecules with different molecular weights. Under SEM condition, the morphology and size of leaf EuRPs were uniform and regular in October, while they were larger morphological difference in samara EuRPs (Fig. 2).

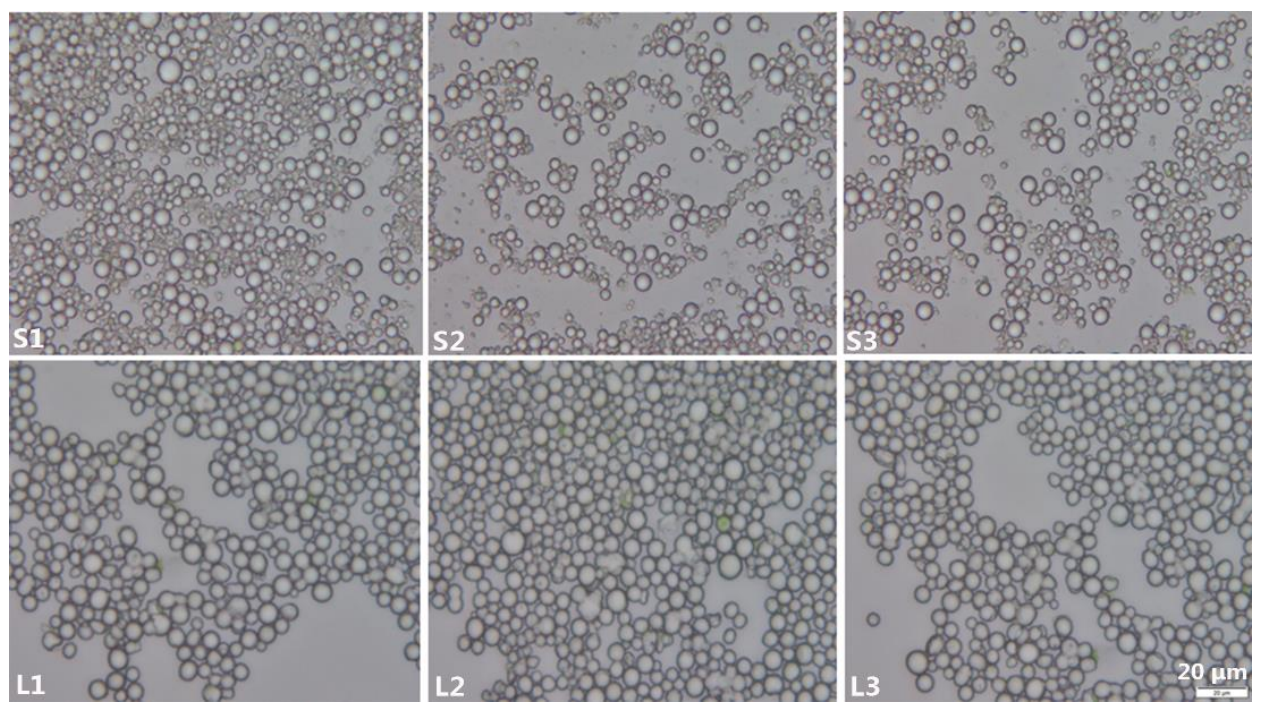

Fig. 1 Optical microscope observation of EuRPs extracted from leaves (L) and samaras (S) 


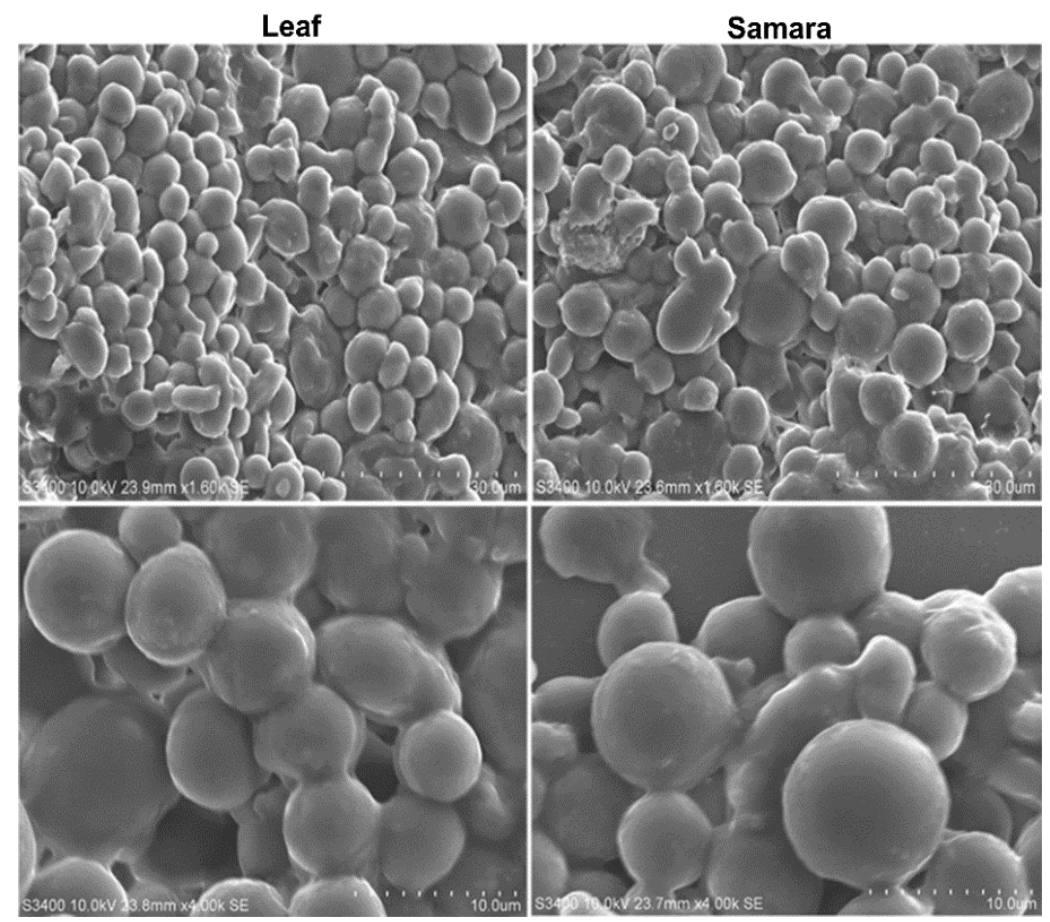

Fig. 2 SEM observation of EuRPs extracted from leaves (L) and samaras (S)

Upper line, EuRPs observation at $500 \times$; Lower line, EuRPs observation $1200 \times$

\section{Analysis of FAs composition in EuRPs}

The EuRPs from E. ulmoides leaves and samaras were subjected to GC analysis in order to determine the fatty acids composition (Table 2, Supplementary Figure 1). Except for myristoleic (C14:1n5), elaidic (C18:1n9t), linoelaidic (C18:2n6t) and arachidic acids (C20:0), most of the 35 FAs were determined in both SaRPs and/or LeRPs. Palmitic (C16:0), palmitoleic (C16:1n7), heptadecanoic (C17:1n7), stearic (C18:0), oleic (C18:1n9c), $\alpha$-linolenic (C18:3n3), twenty-one carbonic (C21:0), eicosadienoic (C20:2), eicotrienoic (C20:3n6), erucic (C22:1n9), tricosanoic (C23:0), docosadienoic (C22:2n6), twenty-four carbonic (C24:0), twenty-two carbohexanthin acids $(\mathrm{C} 22: 6 \mathrm{n} 3)$ were detected in both LeRPs and SaRPs and the content of all these FAs above were significant $(p<0.05)$ or extremely significant $(p<0.01)$ higher in SaRPs than those in LeRPs while the contents of eicosanoic (C20:1), eicotrienoic $(\mathrm{C} 20: 3 \mathrm{n} 3)$ and timnodonic acids $(\mathrm{C} 20: 5 \mathrm{n} 3)$ were significant $(p<0.05)$ or extremely significant $(p<0.01)$ higher in LeRPs than those in SaRPs, as summarized in Table 2. Additionally, three kinds of SFAs including eleven carbonic (C11:0), thirteen carbonic (C13:0), arachidic acids (C20:0) were uniquely detected in LeRPs, while $\gamma$ linolenic acid (C18:3n6) was exclusively found in SaRPs although in very low content, which indicated the $\gamma$-linolenic acid might be a constituent of NRCs of rubber chian in SaRPs. Notably, the seven most important FAs in SaRPs accounted for $95.19 \%$ of the total FAs, while the counterparts accounted for $89.40 \%$, which indicated these FAs might be part of an important branch chain in the EU-rubber molecules in SaRPs and LeRPs. Interestingly, the content of timnodonic acid (C20:5n3) in LeRPs was almost 8.64 folds higher than that in SaRPs.

Table 2 Comparison of fatty acid content between Eucommia samara and Eucommia leaves 


\begin{tabular}{|c|c|c|c|}
\hline NO. & Fatty acid types & Samara $\left(\mathrm{mg} \cdot \mathrm{Kg}^{-1}\right)$ & Leaves $\left(\mathrm{mg} \cdot \mathrm{Kg}^{-1}\right)$ \\
\hline 1 & Octanoic acid (C8:0) & $0.0025 \pm 0.0002$ & $0.0020 \pm 0.0002$ \\
\hline 2 & Decanoic acid (C10:0) & $0.0007 \pm 0.0002$ & $0.0027 \pm 0.0003$ \\
\hline 3 & Eleven carbonic acid (C11:0) & -- & $0.0022 \pm 0.0007$ \\
\hline 4 & Lauric acid (C12:0) & $0.0076 \pm 0.0043$ & $0.0139 \pm 0.0023$ \\
\hline 5 & Thirteen carbonic acid (C13:0) & -- & $0.0014 \pm 0.0004$ \\
\hline 6 & Myristic acid (C14:0) & $0.0769 \pm 0.0019$ & $0.1780 \pm 0.0157$ \\
\hline 7 & Myristoleic acid (C14:1n5) & -- & -- \\
\hline 8 & Fifteen carbonic acid (C15:0) & $0.0104 \pm 0.0023$ & $0.0064 \pm 0.0014$ \\
\hline 9 & Hexadecanoic acid (C15:1n5) & $0.0134 \pm 0.0010$ & $0.0106 \pm 0.0004$ \\
\hline 10 & Palmitic acid (C16:0) & $20.2824 \pm 1.4976$ & $10.4462 \pm 1.0645^{*}$ \\
\hline 11 & Palmitoleic acid (C16:1n7) & $0.4658 \pm 0.0971$ & $0.0336 \pm 0.0013 * *$ \\
\hline 12 & Seventeen carbonic acid (C17:0) & $0.2257 \pm 0.0105$ & $0.1526 \pm 0.0316$ \\
\hline 13 & Heptadecanoic acid (C17:1n7) & $0.1625 \pm 0.0002$ & $0.0144 \pm 0.0034 * *$ \\
\hline 14 & Stearic acid (C18:0) & $9.9599 \pm 0.1206$ & $2.5774 \pm 0.0204 *$ \\
\hline 15 & Elaidic acid (C18:1n9t) & -- & -- \\
\hline 16 & Oleic acid $(\mathrm{C} 18: \ln 9 \mathrm{c})$ & $18.1862 \pm 0.6296$ & $1.0198 \pm 0.0119^{* *}$ \\
\hline 17 & Linoelaidic acid (C18:2n6t) & -- & -- \\
\hline 18 & Linoleic acid (C18:2n6c) & $3.8076 \pm 0.3599$ & $2.7122 \pm 0.1531$ \\
\hline 19 & Arachidic acid (C20:0) & -- & -- \\
\hline 20 & $\gamma$-linolenic acid (C18:3n6) & $0.0067 \pm 0.0015$ & -- \\
\hline 21 & Eicosanoic acid (C20:1) & $1.9717 \pm 0.0898$ & $3.3274 \pm 0.1366^{*}$ \\
\hline 22 & $\alpha$-linolenic acid (C18:3n3) & $0.7134 \pm 0.0510$ & $0.0532 \pm 0.0013^{* *}$ \\
\hline 23 & Twenty-one carbonic acid (C21:0) & $0.2143 \pm 0.0149$ & $0.0219 \pm 0.0023^{* *}$ \\
\hline 24 & Eicosadienoic acid (C20:2) & $0.0377 \pm 0.0001$ & $0.0019 \pm 0.0001 * *$ \\
\hline 25 & Twenty-two carbonic acid (C22:0) & $0.1339 \pm 0.1813$ & $0.1796 \pm 0.0129$ \\
\hline 26 & Eicotrienoic acid (C20:3n6) & $0.0123 \pm 0.0075$ & $0.0020 \pm 0.0001 * *$ \\
\hline 27 & Erucic acid (C22:1n9) & $0.0356 \pm 0.0106$ & $0.0048 \pm 0.0005^{* *}$ \\
\hline 28 & Eicotrienoic acid (C20:3n3) & $0.1237 \pm 0.0117$ & $0.6850 \pm 0.0788^{*}$ \\
\hline 29 & Arachidonic acid (C20:4n6) & $2.1108 \pm 0.3091$ & $3.8642 \pm 0.2489$ \\
\hline 30 & Tricosanoic acid (C23:0) & $0.2177 \pm 0.0040$ & $0.0194 \pm 0.0106^{* *}$ \\
\hline 31 & Docosadienoic acid (C22:2n6) & $0.0467 \pm 0.0109$ & $0.0012 \pm 0.0001 * *$ \\
\hline 32 & Twenty-four carbonic acid (C24:0) & $0.0136 \pm 0.0024$ & $0.0054 \pm 0.0015^{* *}$ \\
\hline 33 & Timnodonic acid (C20:5n3) & $0.2687 \pm 0.0264$ & $2.3224 \pm 0.0811^{* *}$ \\
\hline 34 & Tetracotenoic acid (C24:1n9) & $1.9778 \pm 0.2189$ & $7.3980 \pm 0.1184$ \\
\hline 35 & Twenty-two carbohexanthin acid (C22:6n3) & $0.1554 \pm 0.0020$ & $0.0030 \pm 0.0016^{* *}$ \\
\hline
\end{tabular}

All values rqiresent the average of tiiplicate measurements $(* * p<0.01 ; * p<0.05)$

Analysis of Mw and MWD of Eu-rubber

The determination of molecular weight of Eu-rubber in E. ulmoides leaves and samara in October indicated that there were 5 peaks in the mass distribution of rubber molecules in Eucommia rubber particles in leaves, namely $\mathrm{P}_{1}, \mathrm{P}_{2}, \mathrm{P}_{3}, \mathrm{P}_{4}$ and $\mathrm{P}_{5}$, while the samara showed two to three peaks $\left(\mathrm{P}_{1}, \mathrm{P}_{2}\right.$ or/and $\mathrm{P}_{3}$ ) (Fig. 3). Among them, the maximum molecular weight $(\mathrm{Mw})$ of gutta-percha from Eucommia leaves rubber particles was $60.9 \times 10^{4}$, followed by $1.61 \times 10^{4}$, which were both 
significantly $(p<0.05)$ or extremely significantly $(p<0.01)$ higher than these of Eucommia samara $\left(49.58 \times 10^{4}\right.$ and $\left.0.48 \times 10^{4}\right)$. However, further determination of the average molecular weight among the peaks of $\mathrm{Mw}$ in gutta-percha respectively from E. ulmoides leaves and samara showed that the average molecular weight of gutta-percha in leaves was significantly lower $\left(1.83 \times 10^{4}\right)$ $(\mathrm{P}<0.05)$ than that in E. ulmoides leaves $\left(4.15 \times 10^{4}\right)$, which indicated that the latter contained the polymers with more high molecular weight than the former. Additionally, the analysis based on the molecular weight distribution (MWD, $\mathrm{D}=\mathrm{Mw} / \mathrm{Mn}$ ) of E. ulmoides rubber found the $\mathrm{D}$ value of gutta-percha from E. ulmoides leaves and samaras were 6.76 and 40.61 respectively, which showed that rubber molecules with different molecular weight sizes in the leaves of $E$. ulmoides was more and concentrated distribution while rubber molecules with different molecular weight sizes in the samara of E. ulmoides was less and more dispersed.
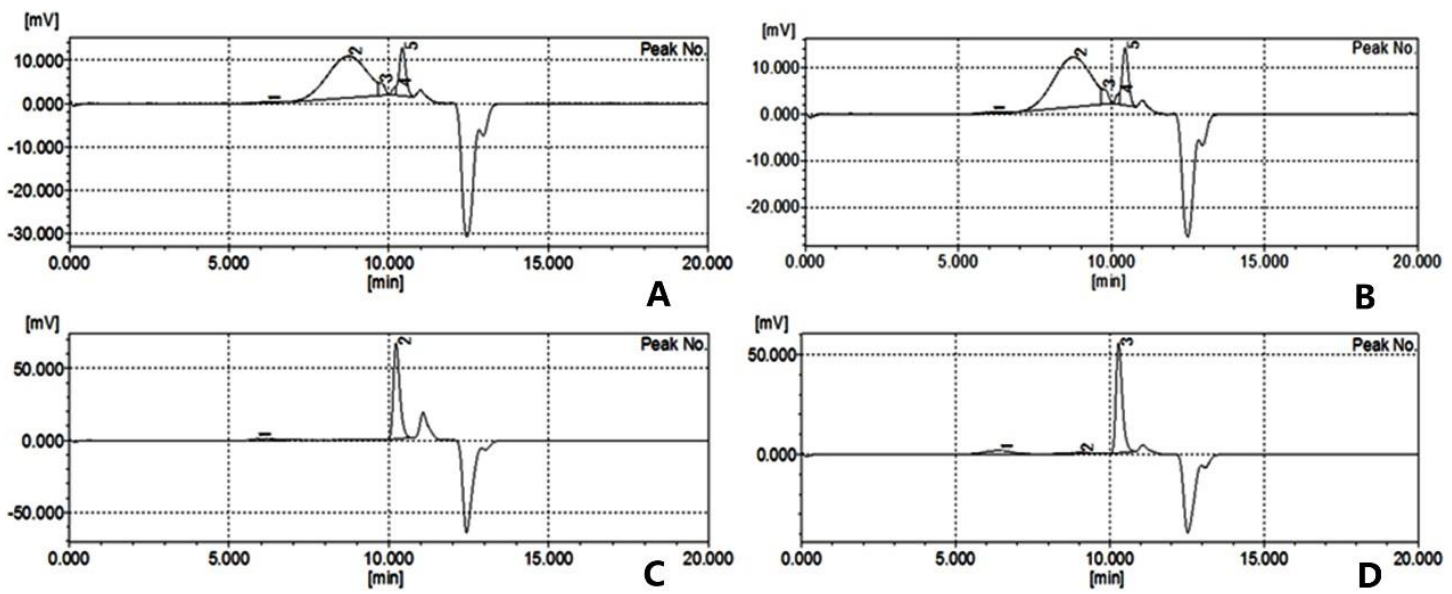

Fig. 3 Molecular-weight distribution of EU-rubber in October

\section{Conclusions}

E. ulmoides Oliver is a woody plant native to China which produces a hard, fibrous long-chain TPI (Nakazawa et al. 2009) and EuRPs (gutta-percha particles) are generally considered to be important sites for E. ulmoides rubber (Wuyun et al. 2018). In this study, we successfully separated EuRPs of E. ulmoides leaves and samaras in October and observed the morphology of gutta-percha particles in virtue of light microscope and electron microscope. The morphology observation of EuRPs extracted from Eucommia ulmoides samara and leaf found that there was little difference in EuRPs size of E. ulmoides leaves and the morphology was more uniform, among which 1-4 $\mu \mathrm{m}$ SRPs accounted for 39\%, 4-7 $\mu \mathrm{m}$ MRPs accounted for 38\%, and 7-11 $\mu \mathrm{m}$ LRPs accounted for $23 \%$, while there was significant differences in EuRPs size of E. ulmoides samas, among which 1-4 $\mu \mathrm{m}$ SRPs accounted for 53\%, 4-7 $\mu \mathrm{m}$ MRPs accounted for 34\%, and 7-11 $\mu \mathrm{m}$ LRPs accounted for $13 \%$. These results suggested that the difference in the distribution of different size particles in different organs and tissues may be related to the specific physiology and metabolism of these PRs in different tissue cells. Studies have shown that the RPs size in various organs of E. ulmoides was different in different periods and increased with the development of organs (Yang et al. 2019). On the one hand, the difference in EuRPs size might reflect differential accumulation of secondary metabolites (e.g. gutta-percha) in the these PRs during the growth and development of the plant organs contained gutta-percha; on the other hand, it also suggested that the difference in protein components (e.g., small rubber particle protein, SRPR) located in the surface of EuRPs which might be involved in the synthesis of gutta-percha with different molecular weight (Xiang et al. 2012; Jing, 2016). 
Fatty acids analysis of leaf EuRPs collected in October by GC-MS method showed that a total of 30 fatty acids were detected in leaf EuRPs and SFAs and UFAs were accounted for $88.3 \%$ and $11.7 \%$ of the total fatty acids determined, respectively, and the results of ANOVA showed that the content difference between them reached a significant level $(\mathrm{P}<0.05)$, which indicated SFAs were the main FAs components in gutta-percha membrane of E. ulmoides leaf EuRPs collected in October. Further study found that the eleven carbonic acid (C11:0) and thirteen carbonic acid (C13:0) were the characteristic FAs in leaves EuRPs of E. ulmoides in October, while the $\gamma$-linolenic acid (C18:3n6) was a unique FA in samaras EuRPs of E. ulmoides. Moreover, the myristoleic acid (C14:1n5), elaidic acid (C18:1n9t) and arachidic acid (C20:0) of 35 fatty acids were detected neither in membrane of leaf EuRPs nor in membrane of samara EuRPs collected in October. Except for a few SFAs, e.g. twenty-one carbonic acid (C21:0), tricosanoic acid (C23:0) and twenty-four carbonic acid (C24:0), the contents of many UFAs including palmitoleic acid (C16:1n7), heptadecanoic acid (C17:1n7), oleic acid (C18:1n9c), $\alpha$-linolenic acid (C18:3n3), eicosadienoic acid (C20:2), eicotrienoic acid (C20:3n6), docosadienoic acid (C22:2n6) and twenty-two carbohexanthin acid $(\mathrm{C} 22: 6 \mathrm{n} 3)$ in plasmalemma of EuRPs isolated from samara were very significant $(\mathrm{p}<0.01)$ higher than these in leaf EuRPs, and the composition of these significantly different fatty acids might indicate the specific function of samara EuRPs in the synthesis of gutta-percha molecules. It is generally believed that UFAs can be inserted into the lipid layer of the plasmalemma, participate in its composition, affect the stability and fluidity of the membrane structure, and are important factors determining the function of the biological membrane ( $\mathrm{Li}$ et al. 2009). The study found that the viscosity of NR was inversely proportional to its lipid content (David et al. 2001). Kakubo et al. (1997) reported the UFAs could reduce the plasticity of NR by being used as plasticizers, on the contrary, some SFAs could influence the crystallization of NR as nucleating agents, and nucleating agents could improve the strength of NR in the unvulcanized state (Kawahara et al. 2011).

The Mw and MWD of Eu-rubber are closely related to the physical properties of gutta-percha, which is an important basis for determining the application range of Eu-rubber (Yang et al. 2019). The use of Eu-rubber with high $\mathrm{Mw}$ is equivalent to that of NR and can be used as a "green tire" and the Eu-rubber with low Mw is characterized by low melting point and strong hardness, which can be used as medically functional materials, shape memory materials and additives (De Barros et al. 1992; Kovuttikulrangsie and Sakdapipanich, 2005). Additionally, Those Eu-rubber with the same $\mathrm{Mw}$ and a narrower MWD have greater strength and plasticity, while other gutta-percha with wider MWD is better elastic, more wear-resistant and heat-resistant (Westall 1968; Guo et al. 2015). GPC analysis of MWD of Eu-rubber molecules in E. ulmoides leaf collected in October showed that there were five characteristic peaks, including high molecular weight ( $\mathrm{HMw})$, medium molecular weight (MMw) and low molecular weight (LMw), while MWD of Eu-rubber molecules in E. ulmoides samara collected at the same month contained two or three characteristic peaks. The average HMw of Eu-rubber molecules from leaf EuRPs and samara EuRPs were $60.9 \times 10^{4}$ and $49.58 \times 10^{4}$, and the $\mathrm{HMw}$ in the former was significantly $(\mathrm{p}<0.05)$ higher than that in the latter. Moreover, the secondary $\mathrm{HMw}$ of Eu-rubber in leaf EuPRs was $1.61 \times 10^{4}$, which it was extremely significant $(p<0.01)$ higher than that of samara EuPRs $\left(0.48 \times 10^{4}\right)$. Among the 5 peaks of MWD of Eu-rubber from leaf EuPRs, the widest peak area was observed in secondary HMw of gutta-percha when it appeared in secondary HMw or third HMw of gutta-percha from samara EuPRs. Compared the secondary HMw or third HMw of MWD of Eu-rubber molecules from samara, the peak area of secondary HMw of Eu-rubber from leaf was much larger than the former, which indicated the latter 
had more gutta-percha molecules with different molecular weights. In leaf EuRPs, the average weight-average molecular weight $(\overline{\mathrm{Mw}})$ of all 5 characteristic peaks of Eu-rubber molecules was $1.83 \times 10^{4}$ while the average number-average molecular weight $(\overline{\mathrm{Mn}})$ was $0.30 \times 10^{4}$, and the value of MWD was the ratio of $\overline{\mathrm{Mw}}$ to $\overline{\mathrm{Mn}}$ (6.1). Synchronously, in samara EuRPs, there were two kinds of average weight-average molecular weight $(\overline{\mathrm{Mw}})$ of all 2-3 characteristic peaks of Eu-rubber molecules, namely $\overline{\mathrm{Mw} 1}$ and $\overline{\mathrm{Mw} 2}$, and there accordingly were two kinds of the average numberaverage molecular weight $(\overline{\mathrm{Mn} 1})$ and $(\overline{\mathrm{Mn}} 2)$. Therefore, the MWD1 and MWD2 were 27.3 and 54.3, respectively. Whatever MWD1 or MWD2 of Eu-rubber in samara EuRPs, both were significant larger than MWD of Eu-rubber in leaf EuRPs (6.1), and the results suggested that there was a larger molecular weight difference of gutta-percha molecules from samara EuRPs while there was a smaller one in gutta-percha molecules from leaf EuRPs. In brief, the Eu-rubber extracted from E. ulmoides leaves in October with characteristics of uniform molecular weight distribution was facilitated to control quality of Eu-rubber and the counterpart from E. ulmoides samaras had excellent processing property but poor uniformity.

\section{Ethics approval and consent to participate}

I certify that the substance of this thesis has not been previously submitted for any other journals. Also, I certify that to the best of my knowledge any help received in preparing this paper and all sources used, have been ackonwledged in this paper.

\section{Consent for publication}

All authors agree to publish the paper.

\section{Availability of data and material}

I guarantee that this article has not been published in any publishing house, nor has anyone's data been stolen.

\section{Competing interests}

All the authors declare that there is no conflict of interest.

\section{Funding}

This study was funded by the National Natural Science Foundation of China (31870285), The "12th Five-Year Plan" for Rural Science and Technology (2013AA102605-05) and Biology First-class Discipline Construction Project of Guizhou Province (2017-009).

\section{Authors' contributions}

In this paper, Lijun Qin is responsible for data analysis, Ting Yuan and Zhengwei Yang are responsible for experimental operation and data collection, and Degang Zhao are responsible for funding.

\section{Acknowledgements}

This work was supported by the National Natural Science Foundation of China (No. 31870285), The "12th Five-Year Plan" for Rural Science and Technology (2013AA102605-05) and Biology First-class Discipline Construction Project of Guizhou Province (GNYL[2017]009). 


\section{Reference}

Bamba T, Fukusaki E, Kajivama S, Ute K, Kitayama T, Kobayashi A (2001) The occurrence of geometric polyprenol isomers in the rubber-producing plant, Eucommia ulmoides oliver. Lipids, 36(7): 727-732.

Chinese Pharmacopoeia Committee of Ministry of Health of the People's Republic of China (1990) Chinese pharmacopoeia. People's Medical Publishing House and Chemical Industry Press, Beijing.

Chollakup R, Tantatherdtam R, Smitthipong W, Rungsanthien K, Suwanruji P, Sriroth K, Radabutra S, Thanawan S, Vallat MF, Nardin M, Mougin K (2013) Effect of non-rubber components on properties of sulphur crosslinked natural rubbers. Advanced Materials Research, 844: 345-348.

Cornish K (2001) Similarities and differences in rubber biochemistry among plant species. Phytochemistry, 57 (7): 1123-1134.

Cronquist A (1981) An integrated system of classification of flowering plants. Columbia University Press, New York.

David MO, Nipithakul T, Nardin M, Schultz J (2001) Influence of non rubber constituent on adhesive properties (tack) of natural rubber. in: The international seminar on elastomer, Le Mans, France.

David MO, Nipithakul T, Nardin M, Schultz J, Suchiva K (2001) Influence of non rubber constituent on adhesive properties (tack) of natural rubber. in: The international seminar on elastomer, Le Mans, France.

De Barros GG, Huang MW, Frisch HL (1992) Interpenetrating polymer networks: high molecular weight natural rubber and poly (2,6-dimethyl-1,4-phenylene oxide). Journal of Applied Polymer Science, 44(2): 255-261.

de Fay E, He' bant Ch, Jacob JL (1989) Cytology and cytochemistry of the laticiferous system. In: d'Auzac J, Jacob J-L, Chrestin H (eds) Physiology of rubber tree latex. CRC Press, Boca Raton, Fla.

Dong J, Ma X, Wei Q, Peng S, Zhang S (2011) Effects of growing location on the contents of secondary metabolites in the leaves of four selected superior clones of Eucommia ulmoides. Industrial Crops and Products, 34(3): 1607-1614.

Guo T, Liu Y, Wei Y, Ma X, Fan Q, Ni J, Yin Z, Liu J, Wang S, Dong Y, Zhang J, Zhang L, Su H, Tan $T$ (2015) Simultaneous qualitation and quantitation of natural trans-1,4-polyisoprene from Eucommia ulmoides Oliver by gel permeation chromatography (GPC). Journal of Chromatography B Analytical Technologies in the Biomedical \& Life Sciences, 1004: 17-22.

Jing T (2016) Study on the function of HMGR, a key enzyme in Eucommia ulmoides rubber synthesis pathway. Beijing: Chinese Academy of Forestry Sciences.

Kakubo T, Matsuura A, Kawahara S, Tanaka Y (1997) Origin of characteristic properties of natural rubber-effect of fatty acids on crystallization of cis-1,4-polyisoprene. Rubber Chemistry and Technology, 71: 70-75.

Kakubo T, Matsuura A, Kawahara S, Tanaka Y (1997) Origin of characteristic properties of natural rubber-effect of fatty acids on crystallization of cis-1,4-polyisoprene. Rubber Chemistry and Technology, 71(1): 70-75.

Kawahara S, Chaikumpollert O, Akabori K, Yamamoto Y (2011) Morphology and properties of natural rubber with nanomatrix of non-rubber components. Polymers Advaced Technologies, 
22(12): 2665-2667.

Kawahara S, Isono I, Kakubo T, Tanaka Y, Eng AH (1999) Crystallization behavior and strength of natural rubber isolated from different Hevea clone. Rubber Chemistry and Technology, 73: 3973.

Kovuttikulrangsie S, Sakdapipanich JT (2005) The molecular weight (MW) and molecular weight distribution (MWD) of NR from different age and clone Hevea trees. Songklanakarin Journal of Science Technology, 27(2): 337-342.

Li XY, Wang JQ, Pu DP, Wei HY, Hu H, Zhou LY (2009) Advanced research of effect of polyunsaturated fatty acids on cell membrane function. Biotechnology Bulletin, 12: 22-26

Nawamawat K, Sakdapipanich JT, Ho CC, Ma Y, Song J, Vancso JG (2011) Surface nanostructure of Hevea brasiliensis natural rubber latex particles. Colloids \& Surfaces A Physicochemical \& Engineering, 390(1-3): 157-166.

Nishiyama N, Kawahara S, Kakubo T, Hwee EA, Tanaka Y (1996) Origin of characteristic properties of natural rubber-synergistic effect of fatty acids on crystallization of cis-1,4polyisoprene: II. Mixed and esterified fatty acids in natural rubber. Rubber Chemistry and Technology, 69: 608-614.

Singh AP, Wi SG, Chuang GC, Kim YS, Kang H (2003). The micromorphology and protein characterization of rubber particles in Ficus carica, Ficus benghalensis and Hevea brasiliensis. Journal of Experimental Botany, 54(384): 985-992.

Sun Z, Li F, Du H, Zhu J, Wang YP (2013) A novel silvicultural model for increasing biopolymer production from Eucommia ulmoides Oliver trees. Industrial Crops and Products, 42: 216-222.

Tarachiwin L, Sakdapipanich J, Ute K, Kitayama T, Bamba T, Fukusaka E, Kobayashi A, Tanaka Y (2005a) Structural characterization of $\alpha$-terminal group of natural rubber. 1. Decomposition of branch-points by lipase and phosphatase treatments. Biomacromolecules, 6: 1851-1857.

Tarachiwin L, Sakdapipanich J, Ute K, Kitayama T, Tanaka Y (2005b) Structural characterization of $\alpha$-terminal group of natural rubber. 2. Decomposition of branch-points by phospholipase and chemical treatments. Biomacromolecules, 6: 1858-1863.

Tarachiwin L, Sakdapipanich JT, Tanaka Y (2005) Relationship between particle size and molecular weight of rubber. Rubber Chemistry and Technology, 78(4): 694-704.

van Beilen JB, Poirier Y (2007) Establishment of new crops for the production of natural rubber. Trends in Biotechnology, 25(11): 522-529.

Westall B (1968) The molecular weight distribution of natural rubber latex. Polymer, 1968, 9: 243248.

Wu LM, Liao SQ, Qu P, Zhou RJ, Wang BX (2014) Structural characterization of natural rubber recent research advancements. 1052: 231-241.

Wu LM, Liao SQ, Qu P, Zhou RJ, Wang BX (2014) Structural characterization of natural rubber recent research advancements. Advanced Materials Research, 1052: 231-241.

Wuyun TN, Wang L, Liu HM , Wang XW, Zhang LS (2018) The hardy rubber tree genome provides insights into the evolution of polyisoprene biosynthesis. Molecular Plant, 11(3): 429-442.

Xiang QL, Dai LJ, Li Y, Nie ZY, Kang GJ, Duan CF, Zeng RZ (2012) Differential centrifugation and differential expression of proteins in rubber particles of rubber tree size. Chinese Agricultural Science Bulletin, 28(04): 18-23.

Yang ZW, Qin LJ, Zhao DG (2019) Extraction, purification and microscopic observation of rubber particles extracted from Eucommia ulmoides. Forest Research, 32(6): 115-121. 
Zhang J, Xue Z (2011) A comparative study on the properties of Eucommia ulmoides gum and synthetic trans-1,4-polyisoprene. Polymer Testing, 30(7): 753-759.

Zhang Z, Zhang H, Turland NJ (2003) Eucommiaceae. In: Flora of China Editorial Committee (eds) Flora of China, vol 9. Science Press, Beijing. 


\section{Figures}
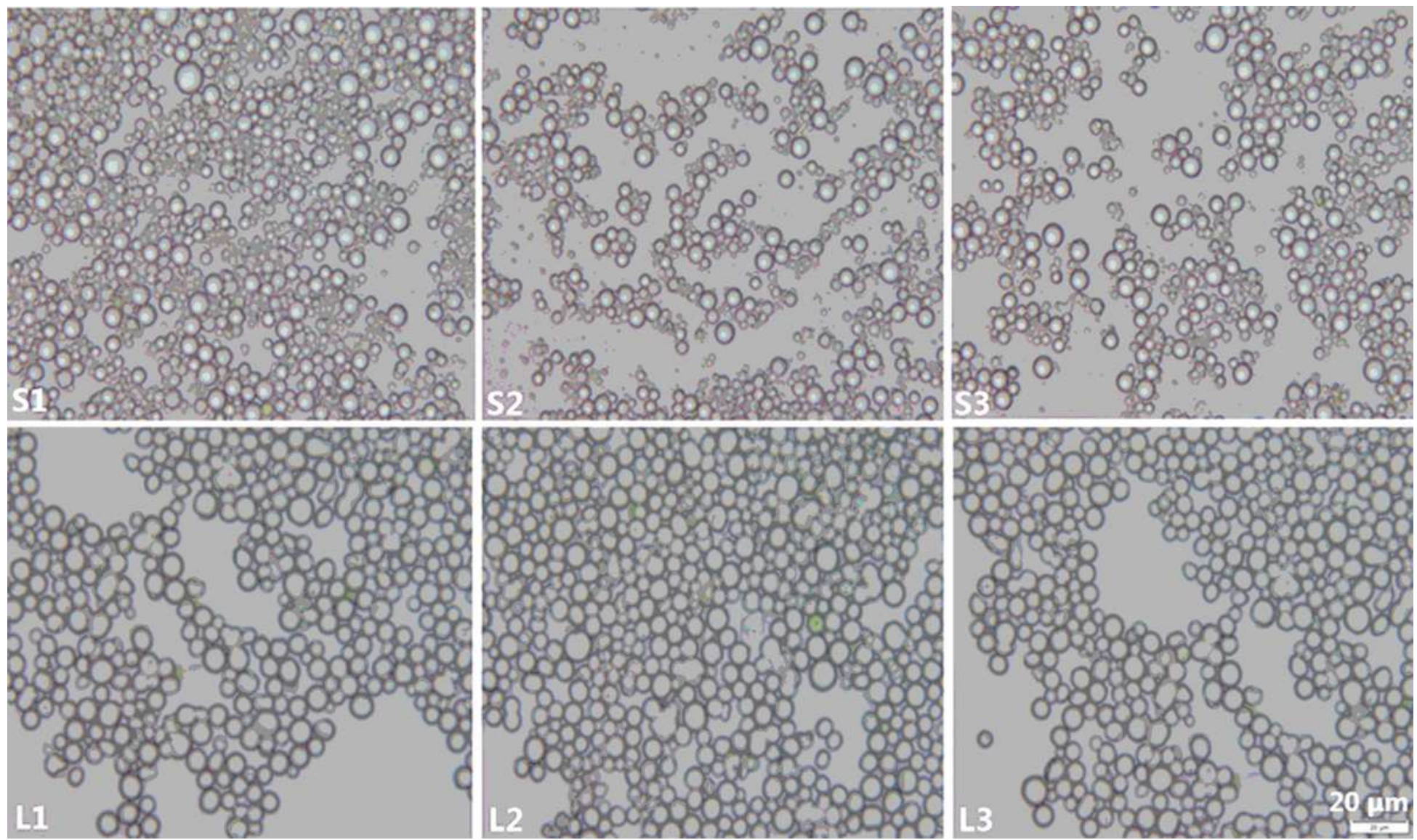

Figure 1

Optical microscope observation of EuRPs extracted from leaves (L) and samaras (S) L1, L2 or L3 S1 and S1, S2 or S3 represented for leaves rubber particles (LeRPs) and samara rubber particles (SaRPs) from 3 independent plants respectively 
Leaf
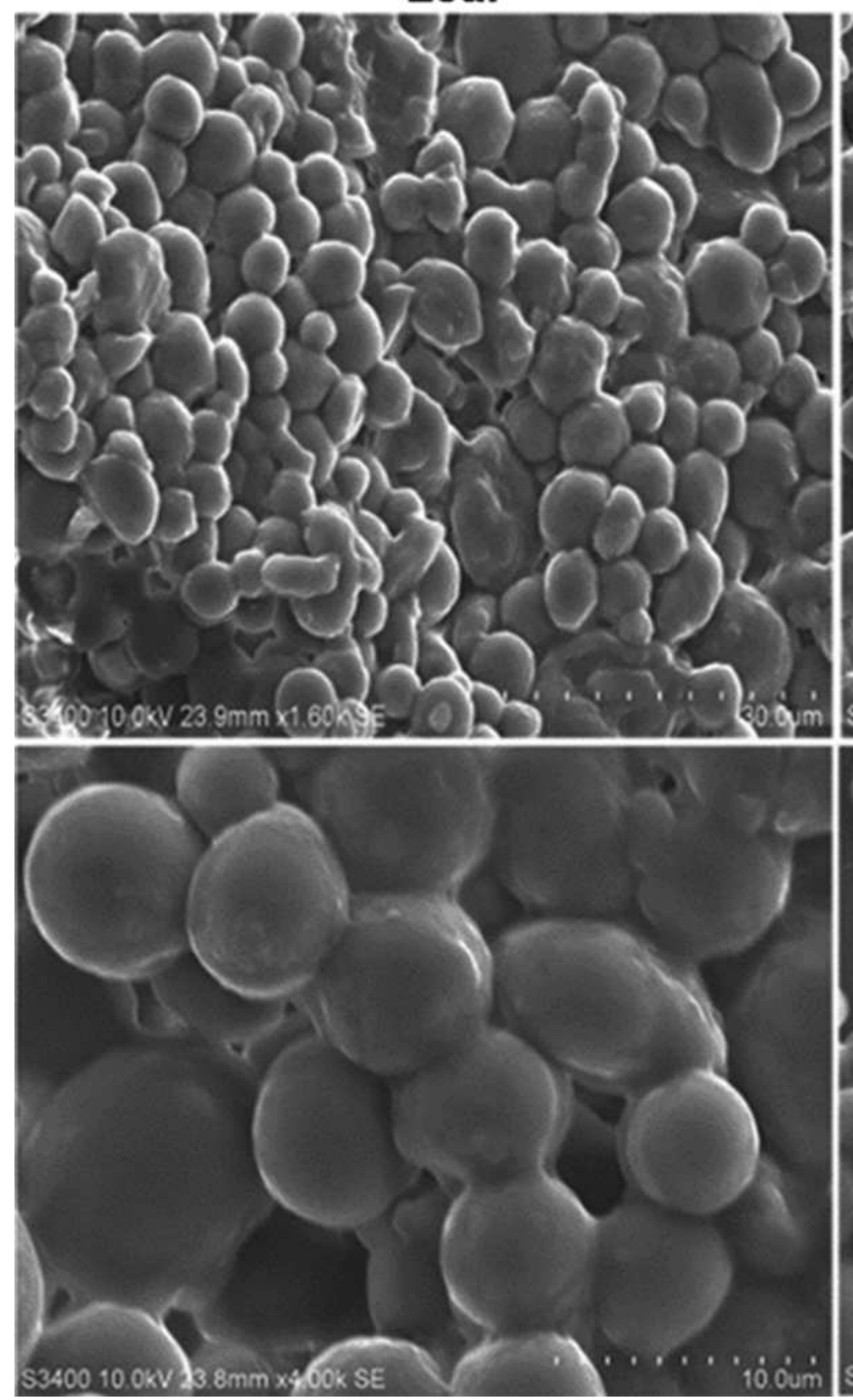

Samara
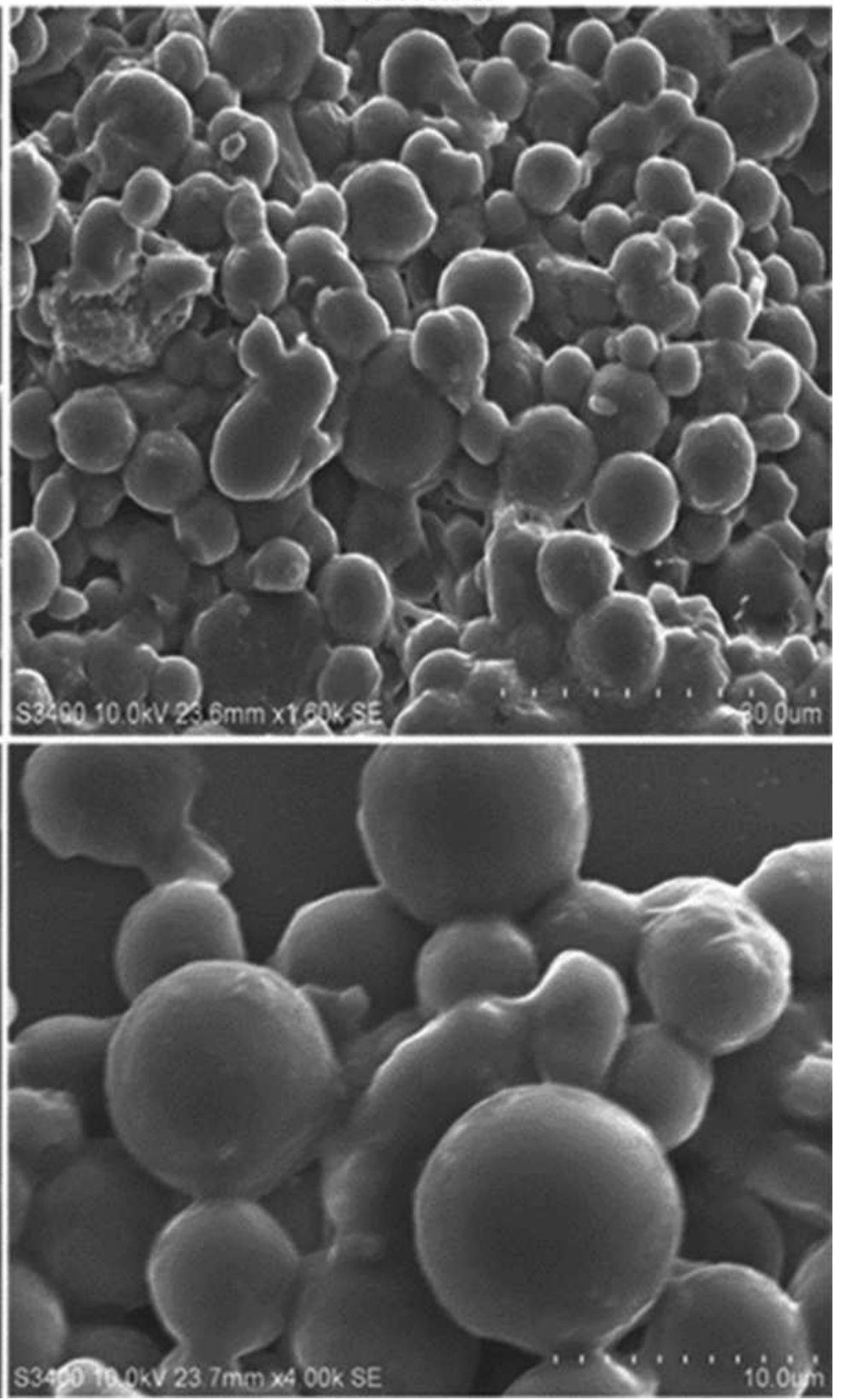

Figure 2

SEM observation of EuRPs extracted from leaves (L) and samaras (S) Upper line, EuRPs observation at 500x; Lower line, EuRPs observation 1200x 

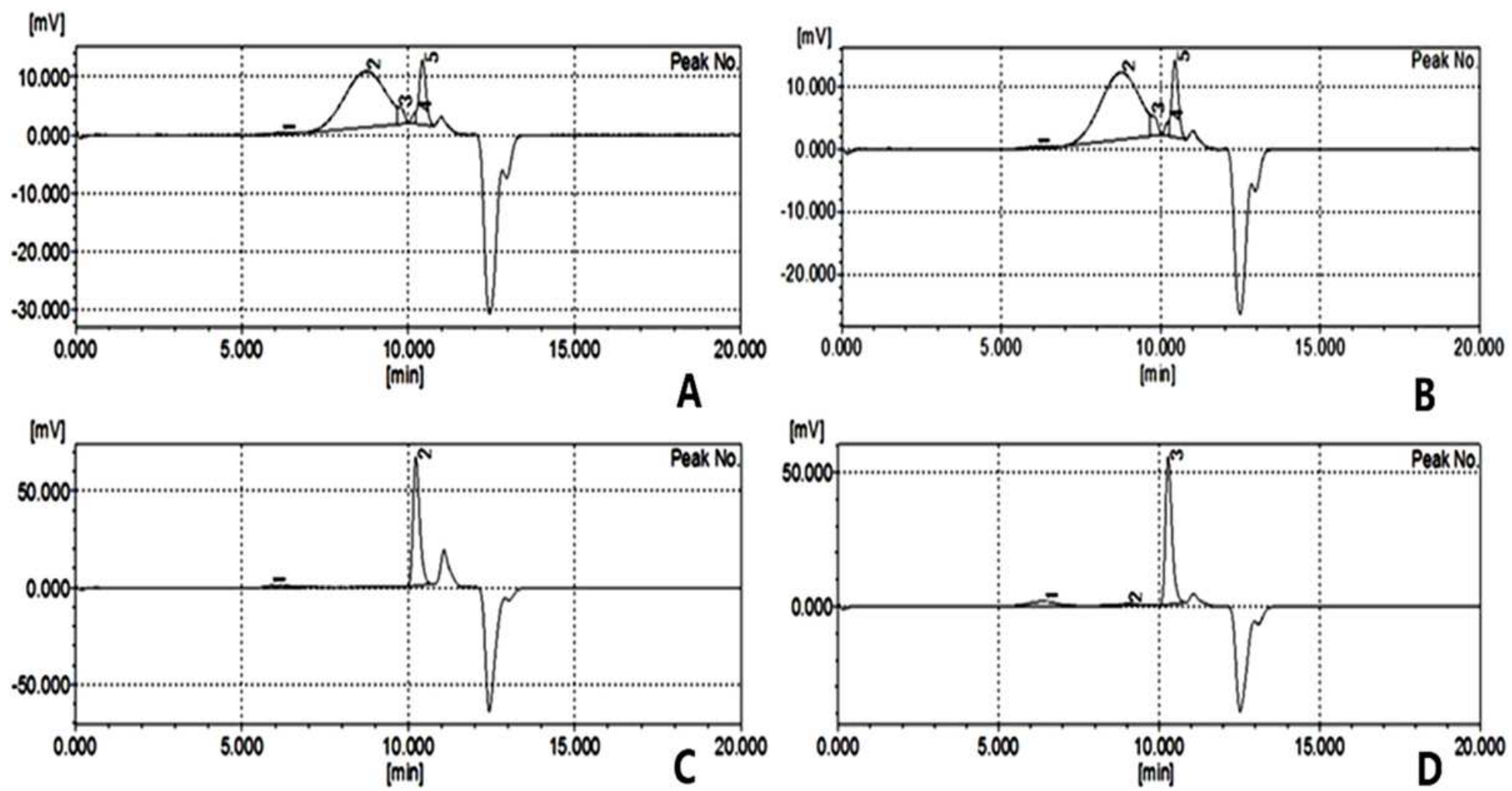

Figure 3

Molecular-weight distribution of EU-rubber in October

\section{Supplementary Files}

This is a list of supplementary files associated with this preprint. Click to download.

- SupplementaryTable.docx

- SupplementaryFigures.docx 